\title{
Dip Emulsion Dosage Form
}

National Cancer Institute

\section{Source}

National Cancer Institute. Dip Emulsion Dosage Form. NCI Thesaurus. Code C149434.

Liquid preparation consisting of an emulsion intended for veterinary use by dipping. 